\title{
Review
}

Journal of Innate

Immunity
J Innate Immun 2011;3:120-130

DOI: $\underline{10.1159 / 000323350}$
Received: November 1, 2010

Accepted after revision: December 6, 2010

Published online: January 14, 2011

\section{The Effect of Bacterial, Viral and Fungal Infection on Mast Cell Reactivity in the Allergic Setting}

\author{
Sarah M. McAlpine Mattias Enoksson Carolina Lunderius-Andersson \\ Gunnar Nilsson \\ Clinical Immunology and Allergy Unit, Department of Medicine, and Centre for Allergy Research, \\ Karolinska Institutet, Stockholm, Sweden
}

\section{Key Words}

Mast cell reactivity $\cdot$ Allergy $\cdot$ Allergic inflammation • Infection

\begin{abstract}
Mast cells are well known for their role in allergic inflammation where, upon aggregation of the high-affinity immunoglobulin E receptor, they release mediators such as histamine that cause classical allergic symptoms. Mast cells are located in almost all tissues and are especially numerous in organs that interface with the environment. Given this strategic location and the more recent notion that they are endowed with receptors that recognize endogenous and exogenous danger signals such as pathogens, it is not surprising that they function as important cells in immune surveillance. When mast cells are activated by pathogens they modulate innate and adaptive immune responses. In allergy, infections might cause exacerbation of the allergic reaction by affecting the reactivity of mast cells. With new developments within the field of mast cell biology, we will better understand how mast cells execute their effector functions. This knowledge will also help to improve the management of allergic diseases.

Copyright $\odot 2011$ S. Karger AG, Basel
\end{abstract}

\section{KARGER}

Fax +4161306 1234

E-Mail karger@karger.ch

www.karger.com

\section{Introduction}

Mast cells are one of our more evolutionary conserved inflammatory cell types, found even in simple organisms such as tunicates [1]. They act as surveillance cells, recognizing intruders or cell injury and rapidly respond by releasing mediators causing vascular effects, recruiting and activating other inflammatory cells, and by modulating an adaptive immune response. Depending on the nature of the receptor-ligand engagement, there will be a differentially regulated mast cell reaction to the insult, and thus, induction of diverse responses dictated by the specific trigger. In allergy, mast cells are infamous for their role as effector cells that cause a harmful reaction through the release of histamine, lipid mediators and cytokines. It is less familiar that mast cells appear to have an important role in host defence to pathogens [2]. Many types of parasites, bacteria, viruses and fungi are recognized by mast cells. This recognition takes place through 2 major ways: either via direct binding to the so-called pattern recognition receptors (PRRs) or via binding of antibody or complement coated bacteria by $\mathrm{Fc}$ receptors or complement receptors on the mast cell surface, respectively. The anti-microbial protective effects of mast cells are, at least 
in part, mediated by their fast release of proteases and cytokines that induce a rapid recruitment of neutrophils to the site of infection [2]. In addition, mast cells might also release antibacterial peptides, such as LL-37 [3], and the formation of extracellular traps [4] that can directly inhibit bacterial growth.

One of the best studied mast cell-mediated reactions is immunoglobulin $\mathrm{E}$ ( $\mathrm{IgE}$ )-dependent mast cell activation where 2 high-affinity IgE receptors, FceRI, are aggregated. How infection affects IgE-mediated mast cell activation is not clear, but it appears that there is a crosstalk between Toll-like receptors (TLRs) and FceRI. There are in vitro studies suggesting that coactivation of TLRs and FceRI augments the release of cytokines from mast cells without affecting the exocytosis of granule mediators [5]. Other studies have shown what appears to be opposite effects where costimulation of TLRs and FceRI inhibits mast cell degranulation [6, 7]. In vivo studies in humans and mice suggest that infections promote the allergic response. Nigo et al. [8] described that lipopolysaccharide (LPS) treatment enhances allergic airway inflammation in a mast cell-dependent fashion. In a human study with rhinovirus, it was demonstrated that infection potentiates histamine release upon allergen provocation in allergic but not in non-allergic subjects [9]. Studies like these indicate that mast cell reactivity is altered by infection and suggest that this might play a role in exacerbations of allergic diseases. In view of the fact that both allergens and microbial antigens can trigger mast cell mediator release and that these triggering pathways are integrated, strategies to modulate these activities might be of therapeutic value. Herein, we will review mast cell biology, their function in host defence and how infection can affect IgE-mediated mast cell activation.

\section{Mast Cell Distribution and Reactivity}

In humans, mast cells are found in almost all tissues and are especially numerous in sites that are in contact with the environment, i.e. skin, respiratory tract, gastrointestinal tract and genitourinary tract. They are divided into 2 major phenotypes: the $\mathrm{MC}_{\mathrm{T}}$ type expressing the protease tryptase and the $\mathrm{MC}_{\mathrm{TC}}$ type containing both tryptase and chymase. The former corresponds to mucosal mast cells in rodents and the latter to the connective tissue mast cells. However, mast cells are a much more heterogeneous cell type than this simple categorization might indicate. Their reactivity to classical mast cell secretagogues, like substance $P$ and compound 48/80, var-

Mast Cell Reactivity to Allergens and Pathogens ies as well as their sensitivity to mast cell inhibitors, such as cromolyn [10]. A recent study of human lung mast cells showed that within the $\mathrm{MC}_{\mathrm{T}}$ and $\mathrm{MC}_{\mathrm{TC}}$ populations, there is great diversity in the expression of different markers related to inflammation [11]. Within the lung, the number of mast cells varies in different compartments, with the small airways and alveolar parenchyma being the sites with the highest mast cell density. In these compartments, the majority of mast cells are of the $\mathrm{MC}_{\mathrm{T}}$ type. In contrast, the mast cell population surrounding pulmonary vessels is composed of approximately $50 \%$ of each $\mathrm{MC}_{\mathrm{T}}$ and $\mathrm{MC}_{\mathrm{TC}}$. Furthermore, the expression of proteins such as FceRI $\alpha$, leukotriene $\mathrm{C}_{4}\left(\mathrm{LTC}_{4}\right)$ synthase, renin and interleukin (IL)-9 varies greatly, not only between $\mathrm{MC}_{\mathrm{T}}$ and $\mathrm{MC}_{\mathrm{TC}}$, but also within these 2 major mast cell phenotypes situated in the different lung compartments [11]. Whether this complex heterogeneity suggests the existence of more mast cell phenotypes than those two that we appreciate today, or it reflects an extended mast cell plasticity dictated by the surrounded cells is not fully known. Since not only the relation between $\mathrm{MC}_{\mathrm{T}}$ and $\mathrm{MC}_{\mathrm{TC}}$ changes with pathogenesis (e.g., in chronic obstructive pulmonary disease) but also the protein expression in the cells differs compared to a healthy lung, it may be that the microenvironment has a great impact on the phenotype of the cells [12].

So far, the expression of TLRs and other receptors of importance for immune surveillance have not been investigated in any detail on specific human mast cell phenotypes or on human mast cells in different tissue compartments. Such studies would be of great interest to better understand the function of mast cells in health and disease. However, the compiled knowledge on receptor expression on mast cells, both human and mouse, as such is rather massive. As discussed in this review, it appears that they have the capacity to express many, if not all, of the crucial receptors for recognizing pathogens.

Receptor-mediated activation of mast cells can have dramatic effects and can ultimately result in an anaphylactic reaction. The most studied and best described activation of mast cells is through the FceRI [13]. Aggregation of FceRI leads to immediate massive exocytosis and release of mediators stored in the granules, including histamine, proteases, heparin and certain cytokines. This is followed within minutes by secretion of leukotrienes and prostaglandins, and some hours thereafter, release of de novo synthesized cytokines, chemokines and growth factors. Of particular interest to understand mast cell biology and the function of this cell in host defence and pathogenesis in different diseases is the differential re- 


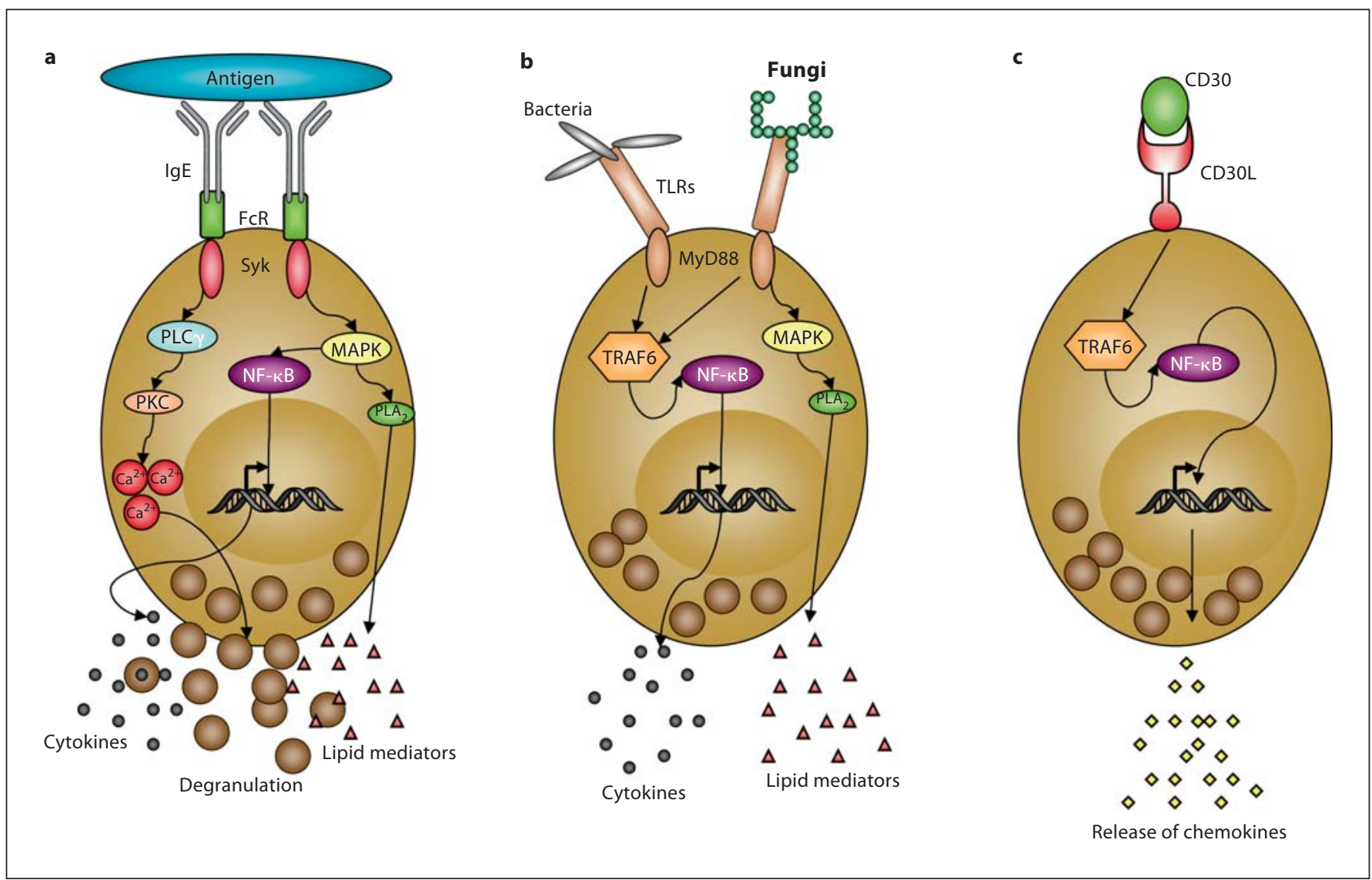

Fig. 1. Activated mast cells release different profiles of mediators depending on the triggering factor. Mast cells can be activated by different means, including for instance FceRI crosslinking (a), TLR activation (b) or CD30-CD30L interaction (c), leading to differential release of mediators. For example, antigens crosslinking FceRI receptors on mast cells induce the activation of 3 separate activation routes, leading to degranulation, release of lipid mediators and de novo production and secretion of cytokines. In contrast, pathogen-derived compounds activating mast cells via

lease of mediators that takes place dependent on the trigger [14] (fig. 1). This means that a trigger acting through FceRI gives rise to degranulation, lipid mediator secretion and release of de novo synthesized cytokines. In contrast, the costimulatory molecule CD30 induces a specific secretion of chemokines, without any preceding degranulation [15]. Most pathogens appear to not induce degranulation, but induce secretion of lipid mediators and de novo synthesis and release of cytokines [16]. Of importance is that different pathogens provoke a specific profile with diverse types of cytokines and/or chemokines released [16]. How the cell is triggered is of great significance for the outcome of the mast cell response.
TLRs do not normally induce degranulation, but instead induce the secretion of lipid mediators and cytokines. Finally, a third response pattern can be observed in mast cells treated with the costimulatory molecule CD30, where mast cells respond by specific secretion of chemokines, but not by degranulation or release of lipid mediators. FcR $=\mathrm{Fc}$ receptor; $\mathrm{PLC} \gamma=$ phospholipase $\mathrm{C} \gamma ; \mathrm{NF}-\kappa \mathrm{B}=$ nuclear factor $-\kappa \mathrm{B} ; \mathrm{MAPK}=$ mitogen-activated protein kinase $\mathrm{PKC}=$ protein kinase $\mathrm{C} ; \mathrm{PLA}_{2}=$ phospholipase $\mathrm{A}_{2}$; TRAF6 = tumour necrosis factor receptor-associated factor 6 .

With the many possibilities, a mast cell-mediated reaction can provoke, including vascular reactions, tissue remodelling, angiogenesis, immunomodulation, cell recruitment, activation of granulocytes and others, it is important to understand that a specific trigger generates a particular set of inflammatory mediators.

\section{Mast Cells Express PRRs}

During evolution, we have been equipped with a variety of defence mechanisms, warding us against the many different types of invading microorganisms. Lacking 
such mechanisms, we would quickly succumb to an overwhelming pathogen burden. Aside from natural barriers like the skin, mucosal surfaces and low gastric $\mathrm{pH}$, we rely on the innate immune system to rapidly detect and eliminate detrimental pathogens. Should a threat overwhelm the innate defence systems, an adaptive immune response can be called upon, thus helping to clear the infection.

The innate branch of the immune system heavily relies on PRRs to detect, identify and neutralize invading pathogens. PRRs are expressed on a variety of cell types, particularly on innate immune cells such as neutrophils, dendritic cells and macrophages, enabling these cell types to rapidly respond to invaders. To combat pathogens, the immune system has evolved several different types of receptors designed to identify microbial species. To date, there are 4 classes of PRRs described: TLRs, C-type lectin receptors (CLRs), Nod-like receptors (NLRs) and retinoic acid-inducible gene-I-like receptors (RLRs). The first TLRs were discovered in the early 1990s, paving the way for the understanding of pathogen recognition. So far, 13 TLRs are identified in humans and 12 in mice [17]. Ligands for the TLRs include bacterial, viral, fungal and also self-components. CLRs seem to, among other functions, have an important role in the defence against fungi, exemplified by the fact that dectin- 1 and dectin- 2 recognize $\beta$-glucan from fungi. NLRs and RLRs recognize bacterial and viral components, respectively, and are present intracellularly, scanning the cytoplasm for microbial components heralding infection.

Together, these 4 classes of receptors, along with their respective subclasses, recognize various and distinct molecular patterns present on microbes, including bacteria, viruses and fungi. Such microbial patterns are generally referred to as pathogen-associated molecular patterns (PAMPs). Although PAMPs come in many different shapes and variants, they are all composed of evolutionary conserved patterns common to certain classes of microbes. Importantly, many PAMPs are composed of microbe components crucial for their viability (e.g., bacterial flagellin and viral single-stranded RNA), thus rendering them more difficult to evolve in a direction that would facilitate immune system evasion. Once detected by PRRs, the presence of PAMPs triggers pathways leading to the induction of pro-inflammatory genes, often through nuclear factor- $\kappa \mathrm{B}$ activation, eventually resulting in the release of pro-inflammatory chemokines and cytokines. Mast cells are counted among the populations expressing different PRRs and are thus able to sense, recognize and respond to microbial intrusions (fig. 2).

Mast Cell Reactivity to Allergens and Pathogens

\section{Mast Cells in Bacterial Infections}

The importance of mast cells in the defence against bacterial infections has been increasingly appreciated during the last decade. Upon bacterial recognition, mast cells can produce pro-inflammatory mediators, including chemokines, thus attracting neutrophils to the site of infection, which will ultimately eliminate the bacteria. In 1996, 2 ground-breaking studies were published providing direct evidence for the importance of mast cells in bacterial infections. In one of these, Echtenacher and colleagues [18] described a protective role for mast cells in acute bacterial peritonitis, assessed using a caecum ligation and puncture (CLP) model. In this model, mast celldeficient $\mathrm{WBB} \mathrm{F}_{1}-K i t^{W / W-v}$ mice displayed a significantly increased mortality rate after CLP, compared to their mast cell-sufficient congenital litter mates. Importantly, mast cell-deficient mice were protected if they were reconstituted intraperitoneally with mast cells prior to CLP.

Another intriguing study was published in the same issue of Nature by Malaviya and coworkers [19]. In their work, mast cell responses to intraperitoneal challenge with enterobacteria were investigated. Interestingly, mast cell-deficient mice were much less efficient in clearing the infection compared to mast cell-sufficient control mice or mast cell-reconstituted mice, again showing that mast cells are important for efficient bacterial defence mechanisms. In addition, mast cells have also been shown to be important for the defence against pathogens such as $M y$ coplasma, Pseudomonas and Helicobater [20-22].

In the studies of Echtenacher et al. [18] and Malaviya et al. [19], mast cells seemed to mediate their protective role by driving tumour necrosis factor (TNF) production upon bacterial encounter, thus attracting neutrophils to the infected peritoneal cavity. It should also be noted that in a more recent study by Piliponsky et al. [23], the importance of mast cells in the survival of mice after CLP was confirmed in a different mast cell-deficient mouse strain, C57BL/6-Kit ${ }^{W-s h / W-s h}$. However, TNF was not important for this mast cell-dependent survival, and in a more severe model of sepsis, the absence of mast cells and mast cell-derived TNF actually increased survival. Therefore, mast cell responses may result in divergent outcomes in various degrees of inflammation, and different models of mast cell-deficient mice may lead to contrasting conclusions in some systems.

A question arising from such studies is how mast cells actually recognize invading bacteria. One answer to this question is that mast cells express different PRRs, allowing them to detect various PAMPs present on bacteria.

J Innate Immun 2011;3:120-130 


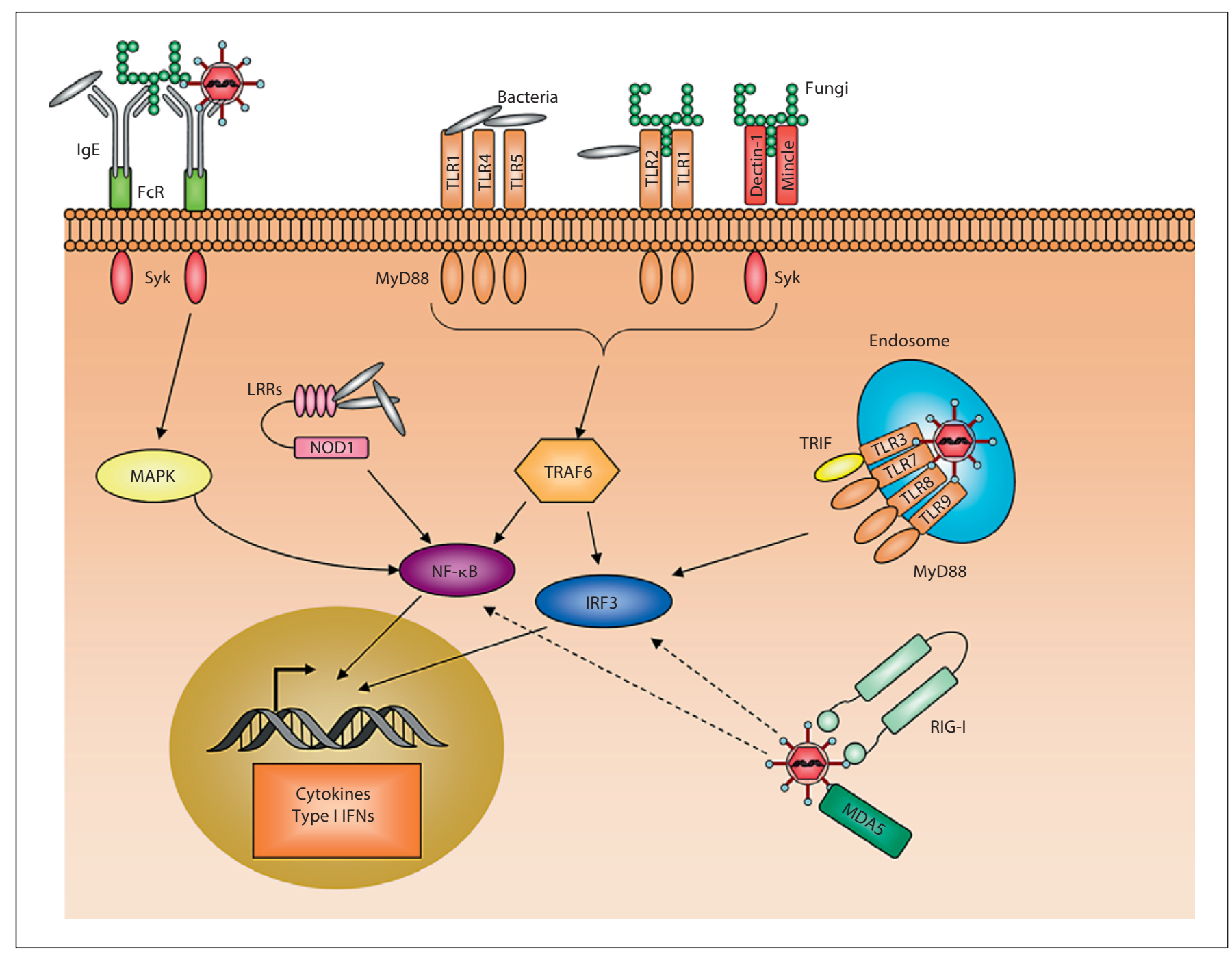

Fig. 2. Mast cells are equipped with a variety of PRRs facilitating their role as sensors of extra- and intracellular pathogens. By expressing extra- and intracellular PRRs such as TLRs, NLRs and CLRs, mast cells can detect and respond to pathogens such as bacteria, viruses and fungi. RLRs may also be expressed by mast cells and enable viral recognition. When activated, mast cells induce differential responses that can include degranulation, lipid mediator release and/or de novo production and release of cytokines and chemokines, depending on the triggering factor. Dashed ar- rows represent hypothetical pathways not yet fully investigated. $\mathrm{FcR}=\mathrm{Fc}$ receptor; $\mathrm{LLRs}=$ lectin-like receptors; $\mathrm{MAPK}=$ mitogenactivated protein kinase; TRAF6 = tumour necrosis factor receptor-associated factor 6 ; NF- $\kappa \mathrm{B}=$ nuclear factor- $\mathrm{kB}$; TRAF $=$ Toll/ IL-1 receptor domain-containing adapter inducing interferon- $\beta$; IRF = interferon regulatory factor; IFNs = interferons; RIG-I = retinoic acid-inducible gene; MDA5 = melanoma differentiationassociated gene 5 .
For instance, it has been shown that TLR4 is important for the protective role played by mast cells in enterobacterial infections. In a study by Supajatura and colleagues [24], it was shown that TLR4 was required for mast cells to respond to LPS (a PAMP present on all Gram-negative bacteria) treatment by secreting cytokines. While mast cells from $\mathrm{C} 3 \mathrm{H} / \mathrm{HeN}$ mice which express functional TLR4 responded to LPS treatment with secretion of sev- eral pro-inflammatory cytokines, mast cells from $\mathrm{C} 3 \mathrm{H} /$ HeJ mice in which TLR4 contains a point mutation and is therefore defective lacked this response. Using a CLP model, the authors could also provide in vivo evidence for the importance of TLR4: mast cell-deficient mice reconstituted with TLR4-mutated mast cells displayed higher mortality compared to mice reconstituted with TLR4-intact mast cells. 
In addition to TLR4, TLR2 has also been shown to be important for the mast cell ability to respond to bacteria. For instance, mast cells derived from TLR2 $2^{+/+}$, but not from $\mathrm{TLR}^{-/-}$mice degranulate in response to peptidoglycan (PGN; a PAMP constituting a part of the bacterial cell wall, especially thick in the cell wall of Gram-positive bacteria) [25]. When injected intradermally in wild-type mice, PGN caused skin reactions manifested due to increased vascular permeability. Interestingly, this response was neither observed in mast cell-deficient mice, nor in mast cell-deficient mice reconstituted with TLR2 ${ }^{-/}$mast cells, suggesting that the vascular permeability was attributed to TLR2-expressing mast cells, which degranulate upon PGN recognition. Taken together, the above mentioned studies provide evidence for the fact that mast cells can detect and respond to bacterial infections, here exemplified by responses elicited towards LPS and PGN, detected by mast cells through TLR4 and TLR2, respectively.

Besides being able to detect bacterial PAMPs through TLRs, data from our group [26] suggest that human cord blood-derived mast cells can respond to the Nod1 agonist M-TriDAP, a PGN derivative, by secretion of pro-inflammatory cytokines and chemokines. Since Nod1 is an intracellular receptor, this suggests that mast cells are also able to detect and respond to intracellular bacteria. Interestingly, the pro-inflammatory response elicited upon MTriDAP treatment could be augmented if M-TriDAP was combined with LPS, suggesting co-operation between intra- and extracellular PRRs on the mast cell.

Furthermore, mouse mast cells also recognize and phagocytose bacteria like Escherichia coli through binding of FimH, a mannose-binding lectin on the fimbriae of bacteria, to CD48 on the mast cell surface $[19,27]$. Another Gram-positive bacteria, Staphylococcus aureus, is often associated with allergic diseases such as atopic dermatitis. The recognition of $S$. aureus by human mast cells appears to be mediated through binding to TLR2 and CD48, leading to the release of IL-8 and TNF [28]. How $S$. aureus infection and mast cell activation affects the course of the allergic disease remains to be elucidated.

Aside from detecting bacteria through PRRs, mast cells also have other means of fighting bacteria, including production and release of antimicrobial peptides. For instance, a study by Di Nardo and colleagues [3] revealed that cathelin-related antimicrobial peptide is important for mast cell antimicrobial activity. When cocultured with group A Streptococcus (GAS), wild-type mast cells were shown to be able to reduce bacterial growth. In contrast, mast cells generated from cathelin-related antimicrobial peptide-deficient mice were approximately $50 \%$ less effective in reducing bacterial growth. Another more recent study investigated the susceptibility of mast cell-deficient C57BL/6-Kit Wsh/Wsh mice to GAS infection in vivo [29]. After subcutaneous injections of GAS, the mice displayed larger lesions and larger lesion bacterial loads compared to wild-type mice. This effect could partly be attributed to a mast cell-derived cathelicidin peptide, providing further evidence for the role of mast cells as responders to bacterial intrusions. Also, the antimicrobial peptide LL-37 has been identified as a means through which mast cells combat infection. It was recently described that human lung mast cells could kill wild-type pneumococci after activation by pneumolysin, but had no effect on pneumolysindeficient Pneumococcus [30]. This was caused by mast cell release of the cathelicidin LL-37, thus exhibiting a direct antimicrobial activity. Finally, von Köckritz-Blickwede et al. [4] have reported that human and mouse mast cells, like neutrophils, can release extracellular structures, which are composed of DNA, histones, tryptase and LL37, which entrap and eventually kill bacteria. It has also been reported that mouse mast cells can act as phagocytes [31], thus killing bacteria within acidified vacuoles, much like professional phagocytes.

Conclusively, numerous previous and recent studies together demonstrate that mast cells constitute an important part of host defence, as they are capable of detecting and also eliminating bacterial threats.

\section{Mast Cells in Viral Infection}

Mast cells have only recently begun to be viewed as players in viral infection, and the beneficial role of mast cells in antiviral immune responses is quite a new area of research. Mast cells have been shown to express multiple PRRs that are activated by viral components. The $2 \mathrm{hu}-$ man cell lines LAD-2 and HMC-1 and primary peripheral blood-derived mast cells express TLR3, TLR7 and TLR9 [32]. These receptors enable mast cells to detect viral infection through the recognition of nucleic acids such as single- and double-stranded RNA and unmethylated CpG DNA sequences [17].

The pro-inflammatory function of virus-associated TLRs may explain why mast cells appear resistant to certain viral infections, as seen in the abortive infection of bone marrow-derived mast cells (BMMCs) with murine cytomegalovirus [33]. The synthetic double-stranded RNA agonist of TLR3, polyinosinic:polycytidylic acid [poly(I:C)], as well as vesicular stomatitis virus, stimu- 
lates mast cell production of interferon- $\alpha$, which is well known to induce an antiviral state in host cells $[32,34]$. In mouse mast cells, poly(I:C) and Newcastle disease virus upregulate the costimulatory molecules CD28 and CD80, which may alter T-cell responses [35]. Newcastle disease virus infection also increases the expression of TLR3 and several key antiviral mediators including interferon- $\beta$, ISG15, CCL4, CCL5 and CXCL10. Furthermore, several studies have shown that mast cells are important for the recruitment of Langerhans cells, CD8 ${ }^{+} \mathrm{T}$ cells and natural killer (NK) cells, when stimulated with the TLR7 ligand imiquimod, the TLR3 ligand poly(I:C) and with double-stranded RNA reovirus, respectively [35-37]. Therefore, mast cells can produce antiviral cytokines and chemoattractants for key antiviral effector cells such as macrophages, NK cells and T cells in viral infection and may be important for directing their migration in the in vivo setting.

Mast cells have also been investigated in the context of dengue virus, which is a single-stranded RNA virus found in tropical areas of the world and is the etiological agent of dengue hemorrhagic fever. There is substantial work to support dengue virus infection via Fc $\gamma$ receptors in human mast cell lines and primary cultures, resulting in higher numbers of infected cells and greater viral replication [38-40]. The fact that little to no dengue virus replication occurs in mast cells in the absence of antidengue virus antibodies, suggests that mast cells may be resistant during primary infections. Mast cells also produce several inflammatory mediators when infected with dengue virus, including the cytokines IL-1 $\beta$, IL- 6 and granulocyte macrophage colony-stimulating factor [40]. These cytokines can effect endothelial activation, fever, plasma cell growth and macrophage differentiation, which are all important components of dengue disease. Additionally, dengue-infected human mast cells produce the chemokines CCL3, CCL4 and CCL5, which are known chemoattractants for monocytes/macrophages, dendritic cells, NK cells and T cells [39]. Clearly, mast cells are sites of productive virus infection and also sources of pro-inflammatory mediators that may contribute to dengue virus pathogenesis or viral clearance.

The initial investigation of mast cell responses to viruses initially began in the 1970s when their involvement in the pathogenesis of asthma was considered. Asthma is an inflammatory disease of the airways induced by hyperresponsiveness to environmental stimuli, resulting in bronchoconstriction, oedema and tissue remodeling. It is widely believed that mast cells play a role in the pathogenesis of asthma, due to the fact that many of their pre- formed and de novo synthesized mediators such as histamine and leukotrienes can induce many asthmatic symptoms [for a review, see ref. 41]. Therefore, in this setting, mast cell responses may be detrimental to the host.

A clear link between viruses and asthma exists. In patients with existing asthma, symptoms are worsened during infection by pathogens such as rhinovirus and respiratory syncytial virus [for a review, see ref. 42]. Evidence of mast cell involvement in this mechanism began with the finding that mediators such as histamine are elevated in patients during virus-induced asthma exacerbations $[43,44]$. Mast cell numbers can also be increased in the lung during viral infection of the airways [45]. Additionally, mast cells can undergo degranulation and histamine release when stimulated with numerous viruses of the airways, including influenza and respiratory syncytial virus [46]. All of these studies support a detrimental role for mast cells in virus-induced asthma exacerbations.

Overall, these studies clearly show that mast cells are armed with the tools necessary to detect and respond to viruses and that they can produce antiviral mediators and activate other arms of the immune system. However, they can also be targeted by multiple viruses, and their responses may in fact be harmful to the host in certain infections. More studies are necessary to truly understand the effect of mast cell responses on the overall clearance of virus and the resolution of infection.

\section{Mast Cell Recognition of Fungi}

Fungi belong to the group of pathogens capable of invading the body through mucosal surfaces and the skin. The fungal intrusion is likely detected through a combination of different receptors; however, today, most of the identified receptors involved in fungal recognition belong to the family of CLRs such as dectin-1, dectin-2, DCSIGN (dendritic cell-specific ICAM-3-grabbing non-integrin), mannose receptor, Mincle receptor and TLR2, TLR4 and TLR9 [17]. Besides being important for the recognition of fungi, CLRs can also recognize other pathogens [47] as well as endogenous danger signals [48].

The function of mast cells in fungal defense is a rather new field of interest where more discoveries still lie ahead. However, recent studies identify mast cells as capable of recognizing fungal products such as zymosan (Saccharomyces cerevisiae) [49], mature fungal hyphae (Aspergillus fumigatus) [50] and also fungal cell extract (Malassezia sympodialis) [51, 52]. Earlier studies have used zymosan; however, not with the focus on the role of mast cells in 
fungal infections, but rather as an inducer of peritonitis and other infectious conditions. Nonetheless, mast cells have been shown to play a role in zymosan-induced peritonitis in the mouse [53, 54].

The fungal intrusion typically activates the innate immune system to bind, phagocytose and kill the fungal cells, in combination with alerting the adaptive immune system to extinguish the fungal infection. The studies so far show that mast cells express receptors involved in fungal recognition such as dectin-1 [49, 51, 55], Mincle [51] and TLRs [52], and activation through these receptors results in production and release of cytokines and chemokines capable of promoting recruitment of inflammatory cells $[49,51,52]$, production of leukotrienes $[49,52]$ and reactive oxygen species generation [55].

In the Northern hemisphere, skin commensal yeast Malassezia species have been isolated as contributing factors for the development of atopic eczema [56], and the involvement of mast cells in this disease raised the question whether they could be activated by the fungus [51, 52]. Both mouse and human mast cells do respond to extract from M. sympodialis [51, 52]. In mouse cells, the fungal extract directly induces release of cysteinyl leukotrienes, and additionally modulates simultaneous IgE receptor-dependent IL-6 production, as well as augments the degranulation of the cells through a TLR2/MyD88dependent route [52]. Surprisingly, IgE sensitization of mouse mast cells leads to upregulated release of leukotrienes together with monocyte chemotactic protein 1 production and degranulation upon stimulation with the fungal extract. Human mast cells generated from atopic eczema patients, as well as from healthy controls, respond to the fungal extract alone, and in combination with activating IgE receptor crosslinking, by producing IL-6 together with IL-8 [51]. Mast cells (from atopic eczema patients and healthy controls) express dectin- 1 and Mincle, as well as TLR2. Of particular interest, mast cells constitute the majority of the dectin-1-expressing cells in atopic eczema skin [51].

Fungi have for some time been considered to affect atopic diseases, either as a source of allergens or simply by augmenting immune reactions through increased production of proinflammatory cytokines and bronchoconstricting leukotrienes [57]. Furthermore, mast cells generated from patients with atopic eczema lack the ability to upregulate dectin-1 messenger RNA upon stimulation with Malassezia cell extract. This deficiency could reflect malfunctioning receptor(s) in atopic eczema patients causing hampered defense and difficulties to fight off commensal pathogens, eventually leading to perpet-

Mast Cell Reactivity to Allergens and Pathogens ual immune frustration [51]. Additionally, A. fumigatus induces IgE-independent degranulation of mouse mast cells, and may worsen chronic lung diseases [50].

\section{The Effect of TLR Activation on Mast Cell Reactivity to Fce RI Crosslinking}

The signalling pathways induced by ligation of TLRs and FceRI are distinct, involving different adaptor and signalling molecules (fig. 2). Therefore, each activation event results in diverse mediator production. For example, human mast cell cytokine responses to poly(I:C) are distinct from FceRI crosslinking in that TNF is not produced [32]. However, it is very likely that the receptors and mediators upregulated in response to ligands such as PAMPs may alter the result of IgE-mediated mast cell activation.

The majority of the research in this area has focused on bacterial products and cell wall constituents. LPS synergistically enhances BMMC production of the proinflammatory cytokines IL-6, IL-13 and TNF in response to IgE-mediated activation, with no alteration in degranulation or arachidonic acid metabolites [5]. The enhanced cytokine production was shown to be caused by a synergistic activation of mitogen-activated protein kinases, particularly JNK [5]. Furthermore, in vivo, cotreatment with LPS and antigen induces greater production of IL-5 and eotaxin compared to FceRI-crosslinking alone [8]. These findings may have implications for antibody production and eosinophil recruitment, which could negatively impact the allergic condition. Surprisingly, IgEsensitized mouse mast cells treated with LPS in the absence of antigen are also synergistically activated to produce IL- 6 and TNF, suggesting that sensitized individuals may experience greater inflammation during bacterial infection than those who are not sensitized [58]. IgE and LPS costimulation can also rescue BMMCs from apoptosis due to IL-3 withdrawal, meaning that mast cells may survive longer in sensitized patients as well [59].

TLR2 recognizes a wide range of bacterial products and components and is expressed on human mast cells [60]. The TLR2 ligand PGN acts similarly to LPS by synergistically acting with FceRI signalling to upregulate IL- 6 and TNF production in BMMCs [5]. This synergism appears to be a consistent response to bacterial products, as the TLR2 agonist $\mathrm{Pam}_{3} \mathrm{CSK}_{4}$ lipopeptide also enhances IL-6 production during FceRI crosslinking [6]. However, not all IgE-mediated mast cell responses are augmented by TLR 2 activation. Mast cell degranulation is 
inhibited by the TLR2 agonists PGN, lipoteichoic acid [7] and $\mathrm{Pam}_{3} \mathrm{CSK}_{4}$ [6]. This inhibitory effect has also been observed after stimulation of the rat cell line RBL-2H3 with the Gram-positive bacterium Bifidobacterium [61]. More importantly, $\mathrm{Pam}_{3} \mathrm{CSK}_{4}$ also inhibits the production of classical FceRI-driven mediators such as IL-13 and $\mathrm{LTC}_{4}$, suggesting that the recognition of bacterial infection may prevent IgE isotype switching, mucus and collagen production, as well as bronchoconstriction.

All of these studies combined provide strong evidence for an interaction between FceRI and TLR signalling in mast cells. However, considering the contrasting effects of TLR2 and TLR4 stimulation, the overall outcome of mast cell responses to bacterial infection is likely to be highly dependent on the nature of the bacterial stimulus, and on the combination of signals delivered to the mast cell.

Very few studies in this area have focused on pathogens other than bacteria. Interestingly, low doses of extract from the skin commensal yeast Malassezia can augment IL- 6 production by mouse mast cells, while high doses can inhibit this response, and both responses are dependent on TLR2 [52]. This perhaps indicates that mediator production by mast cells may be affected by the level of infection, and therefore, the potency of the stimulus. This yeast extract can also enhance degranulation driven by FceRI crosslinking; however, these responses are TLR2 and MyD88 independent. In the class of parasites, filarial nematodes produce an anti-inflammatory glycoprotein called ES-62, which can bind to and activate TLR4 [62]. ES-62 inhibits the amount of degranulation and $\mathrm{LTC}_{4}$ and prostaglandin $\mathrm{D}_{2}$ production by FceRIcrosslinked human mast cells. This may lead to an inhibition of allergic-type responses in parasite-infected patients and may also have detrimental effects on pathogen clearance, since Th2 responses are so important for immunity to parasites.

A study by Kulka et al. [32] demonstrated that the viral double-stranded RNA analogue poly(I:C) pretreatment inhibits human mast cell degranulation induced by FceRI crosslinking in an in vitro adhesion model. This finding suggests that poly(I:C) recognition may reduce allergic responses and is in contradiction to previously mentioned studies showing that viral infection can induce mast cell degranulation $[46,63]$. This may be explained by the simplicity of poly(I:C) as a model for viral infection. Alternatively, it may indicate that mast cell responses to viral infection are largely determined by localization of the pathogen, where infection of neighbouring cells, as detected by the uptake of double-stranded RNA into the
TLR3-containing endosomal compartment, induces an appropriate response compared to infection of the mast cell itself. Moreover, infections can directly induce IgEmediated responses through the binding of superantigens to IgE on the human mast cell surface, thereby crosslinking the IgE receptors and release of mediators [64]. Examples of such superantigens are protein Fv, an endogenous protein synthesized in the human liver and increased during viral hepatitis, protein A of S. aureus and the envelope glycoprotein gp120 of HIV-1.

\section{Conclusion}

For many years, mast cells were more or less ignored, except by allergologists, since they were merely considered to be effector cells of allergic inflammation. During the last 2 decades, this view has dramatically changed. Today, we know that mast cells play an important role in both the initiation of an innate immune response and in the coordination of adaptive immune responses. This knowledge about mast cell biology has drawn attention to the role of mast cells in the pathogenesis of many diseases, beyond allergic inflammation, and also to the critical role of mast cells in host defence. The ability of mast cells to react to both harmless agents such as allergens and harmful pathogens might cause problems in allergic diseases, such as atopic eczema and asthma, where exacerbations are common in conjunction with infections. By furthering investigations into the role of mast cells in innate and adaptive immune responses, and the effect of infections on their reactivity, we will better understand the multifunctional role of mast cells in health and disease. Hopefully, this will also lead to new strategies to reduce allergic symptoms.

\section{Acknowledgements}

G.N. is supported by the Swedish Research Council and the Swedish Heart Lung Foundation. S.M.M. was awarded an HQP exchange award, supported by the International Partnership Initiative between AllerGen NCE Inc. and the Centre for Allergy Research at the Karolinska Institutet. 


\section{References}

$>1$ Crivellato E, Ribatti D: The mast cell: an evolutionary perspective. Biol Rev Camb Philos Soc 2010;85:347-360.

-2 Abraham SN, St John AL: Mast cell-orchestrated immunity to pathogens. Nat Rev Immunol 2010;10:440-452.

$>3$ Di Nardo A, Vitiello A, Gallo RL: Cutting edge: mast cell antimicrobial activity is mediated by expression of cathelicidin antimicrobial peptide. J Immunol 2003;170:22742278.

4 von Köckritz-Blickwede M, Goldmann O, Thulin P, Heinemann K, Norrby-Teglund A, Rohde M, Medina E: Phagocytosis-independent antimicrobial activity of mast cells by means of extracellular trap formation. Blood 2008;111:3070-3080.

5 Qiao H, Andrade MV, Lisboa FA, Morgan K, Beaven MA: FcepsilonR1 and Toll-like receptors mediate synergistic signals to markedly augment production of inflammatory cytokines in murine mast cells. Blood 2006; 107:610-618

6 Fehrenbach K, Port F, Grochowy G, Kalis C, Bessler W, Galanos C, Krystal G, Freudenberg M, Huber M: Stimulation of mast cells via FcvarepsilonR1 and TLR2: the type of ligand determines the outcome. Mol Immunol 2007;44:2087-2094.

>7 Yoshioka M, Fukuishi N, Iriguchi S, Ohsaki $\mathrm{K}$, Yamanobe $\mathrm{H}$, Inukai A, Kurihara D, Imajo N, Yasui Y, Matsui N, Tsujita T, Ishii A, Seya T, Takahama M, Akagi M: Lipoteichoic acid downregulates FcepsilonRI expression on human mast cells through Toll-like receptor 2. J Allergy Clin Immunol 2007;120: 452-461.

-8 Nigo YI, Yamashita M, Hirahara K, Shinnakasu R, Inami M, Kimura M, Hasegawa A, Kohno Y, Nakayama T: Regulation of allergic airway inflammation through Toll-like receptor 4-mediated modification of mast cell function. Proc Natl Acad Sci USA 2006;103: 2286-2291.

9 Calhoun WJ, Dick EC, Schwartz LB, Busse WW: A common cold virus, rhinovirus 16 , potentiates airway inflammation after segmental antigen bronchoprovocation in allergic subjects. J Clin Invest 1994;94:22002208.

10 Nilsson G, Schwartz LB: Mast cell heterogeneity: structure and mediators; in Busse WW, Holgate ST (eds): Asthma and Rhinitis. Boston, Blackwell Science, 1995, pp 195-208.

-11 Andersson CK, Mori M, Bjermer L, Lofdah. CG, Erjefalt JS: Novel site-specific mast cell subpopulations in the human lung. Thorax 2009;64:297-305

$\checkmark 12$ Andersson CK, Mori M, Bjermer L, Lofdahl CG, Erjefalt JS: Alterations in lung mast cell populations in patients with chronic obstructive pulmonary disease. Am J Respir Crit Care Med 2010;181:206-217.
13 Gilfillan AM, Rivera J: The tyrosine kinase network regulating mast cell activation. Immunol Rev 2009;228:149-169.

14 Theoharides TC, Kempuraj D, Tagen M, Conti P, Kalogeromitros D: Differential release of mast cell mediators and the pathogenesis of inflammation. Immunol Rev 2007;217:65-78.

15 Fischer M, Harvima IT, Carvalho RFS, Moller C, Naukkarinen A, Enblad G, Nilsson $\mathrm{G}$ : Mast cell CD30 ligand is upregulated in cutaneous inflammation and mediates degranulation-independent chemokine secretion. J Clin Invest 2006;116:2748-2756.

16 Marshall JS: Mast-cell responses to pathogens. Nat Rev Immunol 2004;4:787-799.

17 Takeuchi O, Akira S: Pattern recognition receptors and inflammation. Cell 2010;140: 805-820.

18 Echtenacher B, Mannel DN, Hultner L: Critical protective role of mast cells in a model of acute septic peritonitis. Nature 1996;381:7577.

19 Malaviya R, Ikeda T, Ross E, Abraham SN: Mast cell modulation of neutrophil influx and bacterial clearance at sites of infection through TNF-alpha. Nature 1996;381:7780.

20 Siebenhaar F, Syska W, Weller K, Magerl M, Zuberbier T, Metz M, Maurer M: Control of Pseudomonas aeruginosa skin infections in mice is mast cell-dependent. Am J Pathol 2007;170:1910-1916.

21 Velin D, Bachmann D, Bouzourene H, Michetti P: Mast cells are critical mediators of vaccine-induced Helicobacter clearance in the mouse model. Gastroenterology 2005; 129:142-155.

22 Xu X, Zhang D, Lyubynska N, Wolters PJ, Killeen NP, Baluk P, McDonald DM, Hawgood S, Caughey GH: Mast cells protect mice from mycoplasma pneumonia. Am J Respir Crit Care Med 2006;173:219-225.

23 Piliponsky AM, Chen CC, Grimbaldeston MA, Burns-Guydish SM, Hardy J, Kalesnikoff J, Contag CH, Tsai M, Galli SJ: Mast cell-derived TNF can exacerbate mortality during severe bacterial infections in C57BL/6-KitW-sh/W-sh mice. Am J Pathol 2010;176:926-938.

24 Supajatura V, Ushio H, Nakao A, Okumura $\mathrm{K}$, Ra C, Ogawa H: Protective roles of mast cells against enterobacterial infection are mediated by Toll-like receptor 4 . J Immunol 2001;167:2250-2256.

25 Supajatura V, Ushio H, Nakao A, Akira S, Okumura K, Ra C, Ogawa H: Differential responses of mast cell Toll-like receptors 2 and 4 in allergy and innate immunity. J Clin Invest 2002;109:1351-1359.
26 Enoksson M, Ejendal KFK, McAlpine S, Nilsson G, Lunderius-Andersson C: $\mathrm{Hu}-$ man cord blood-derived mast cells are activated by the Nod1 agonist M-TriDAP to release pro-inflammatory cytokines and chemokines. J Innate Immun 2011;3:142149.

27 Malaviya R, Gao Z, Thankavel K, van der Merwe PA, Abraham SN: The mast cell tumor necrosis factor alpha response to FimHexpressing Escherichia coli is mediated by the glycosylphosphatidylinositol-anchored molecule CD48. Proc Natl Acad Sci USA 1999; 96:8110-8115.

28 Rocha-de-Souza CM, Berent-Maoz B, Mankuta D, Moses AE, Levi-Schaffer F: Human mast cell activation by Staphylococcus aureus: interleukin-8 and tumor necrosis factor alpha release and the role of Toll-like receptor 2 and CD48 molecules. Infect Immun 2008;76:4489-4497.

29 Di Nardo A, Yamasaki K, Dorschner RA, Lai Y, Gallo RL: Mast cell cathelicidin antimicrobial peptide prevents invasive group A Streptococcus infection of the skin. J Immunol 2008;180:7565-7573.

-30 Cruse G, Fernandes VE, de Salort J, Pankhania D, Marinas MS, Brewin H, Andrew PW, Bradding P, Kadioglu A: Human lung mast cells mediate pneumococcal cell death in response to activation by pneumolysin. J Immunol 2010;184:7108-7115.

31 Malaviya R, Ross EA, MacGregor JI, Ikeda T, Little JR, Jakschik BA, Abraham SN: Mast cell phagocytosis of FimH-expressing enterobacteria. J Immunol 1994;152:19071914.

32 Kulka M, Alexopoulou L, Flavell RA, Metcalfe DD: Activation of mast cells by doublestranded RNA: evidence for activation through Toll-like receptor 3. J Allergy Clin Immunol 2004; 114:174-182.

-33 Gibbons AE, Price P, Robertson TA, Papadimitriou JM, Shellam GR: Replication of murine cytomegalovirus in mast cells. Arch Virol 1990;115:299-307.

34 Dietrich N, Rohde M, Geffers R, Kroger A, Hauser H, Weiss S, Gekara NO: Mast cells elicit proinflammatory but not type I interferon responses upon activation of TLRs by bacteria. Proc Natl Acad Sci USA 2010;107: 8748-8753.

35 Orinska Z, Bulanova E, Budagian V, Metz M, Maurer M, Bulfone-Paus S: TLR3-induced activation of mast cells modulates CD8+ Tcell recruitment. Blood 2005;106:978-987.

-36 Burke SM, Issekutz TB, Mohan K, Lee PW, Shmulevitz M, Marshall JS: Human mast cell activation with virus-associated stimuli leads to the selective chemotaxis of natural killer cells by a CXCL8-dependent mechanism. Blood 2008;111:5467-5476. 
-37 Heib V, Becker M, Warger T, Rechtsteiner G, Tertilt C, Klein M, Bopp T, Taube C, Schild $\mathrm{H}$, Schmitt E, Stassen M: Mast cells are crucial for early inflammation, migration of Langerhans cells, and CTL responses following topical application of TLR7 ligand in mice. Blood 2007;110:946-953.

38 Brown MG, King CA, Sherren C, Marshall JS, Anderson R: A dominant role for FcgammaRII in antibody-enhanced dengue virus infection of human mast cells and associated CCL5 release. J Leukoc Biol 2006;80:12421250.

39 King CA, Anderson R, Marshall JS: Dengue virus selectively induces human mast cell chemokine production. J Virol 2002;76: 8408-8419.

-40 King CA, Marshall JS, Alshurafa H, Anderson R: Release of vasoactive cytokines by antibody-enhanced dengue virus infection of a human mast cell/basophil line. J Virol 2000; 74:7146-7150.

-41 Okayama Y, Kirshenbaum AS, Metcalfe DD: Expression of a functional high-affinity IgG receptor, Fc gamma RI, on human mast cells: up-regulation by IFN-gamma. J Immunol 2000;164:4332-4339.

42 Kumar A, Grayson MH: The role of viruses in the development and exacerbation of atopic disease. Ann Allergy Asthma Immunol 2009; 103:181-186, quiz 186-187, 219.

$\checkmark 43$ Calhoun WJ, Swenson CA, Dick EC, Schwartz LB, Lemanske RF Jr, Busse WW: Experimental rhinovirus 16 infection potentiates histamine release after antigen bronchoprovocation in allergic subjects. Am Rev Respir Dis 1991;144:1267-1273.

44 Oymar K, Halvorsen T, Aksnes L: Mast cell activation and leukotriene secretion in wheezing infants. Relation to respiratory syncytial virus and outcome. Pediatr Allergy Immunol 2006; 17:37-42.

-45 Castleman WL, Sorkness RL, Lemanske RF Jr, McAllister PK: Viral bronchiolitis during early life induces increased numbers of bronchiolar mast cells and airway hyperresponsiveness. Am J Pathol 1990;137:821-831.
46 Ogunbiyi PO, Black WD, Eyre P: Parainfluenza-3 virus-induced enhancement of histamine release from calf lung mast cells - effect of levamisole. J Vet Pharmacol Ther 1988;11: 338-344

47 Geijtenbeek TB, Gringhuis SI: Signalling through C-type lectin receptors: shaping immune responses. Nat Rev Immunol 2009;9: 465-479.

48 Yamasaki S, Ishikawa E, Sakuma M, Hara H, Ogata K, Saito T: Mincle is an ITAM-coupled activating receptor that senses damaged cells. Nat Immunol 2008;9:1179-1188.

49 Olynych TJ, Jakeman DL, Marshall JS: Fungal zymosan induces leukotriene production by human mast cells through a dectin-1-dependent mechanism. J Allergy Clin Immunol 2006;118:837-843.

50 Urb M, Pouliot P, Gravelat FN, Olivier M, Sheppard DC: Aspergillus fumigatus induces immunoglobulin E-independent mast cell degranulation. J Infect Dis 2009;200:464472.

51 Ribbing C, Engblom C, Lappalainen J, Lindstedt K, Kovanen PT, Karlsson MA, Lundeberg L, Johansson C, Nilsson G, LunderiusAndersson C, Scheynius A: Mast cells generated from patients with atopic eczema have enhanced levels of granule mediators and an impaired dectin-1 expression. Allergy 2011; 66:110-119.

52 Selander C, Engblom C, Nilsson G, Scheynius A, Andersson CL: TLR2/MyD88-dependent and -independent activation of mast cell IgE responses by the skin commensal yeast $\mathrm{Mal}$ assezia sympodialis. J Immunol 2009; 182 : 4208-4216.

53 Kolaczkowska E, Lelito M, Kozakiewicz E, van Rooijen N, Plytycz B, Arnold B: Resident peritoneal leukocytes are important sources of MMP-9 during zymosan peritonitis: superior contribution of macrophages over mast cells. Immunol Lett 2007;113:99-106.

54 Mullaly SC, Kubes P: Mast cell-expressed complement receptor, not TLR2, is the main detector of zymosan in peritonitis. Eur J Immunol 2007;37:224-234.

55 Yang Z, Marshall JS: Zymosan treatment of mouse mast cells enhances dectin-1 expression and induces dectin-1-dependent reactive oxygen species (ROS) generation. Immunobiology 2009;214:321-330.
56 Scheynius A, Johansson C, Buentke E, Zargari A, Linder MT: Atopic eczema/dermatitis syndrome and Malassezia. Int Arch Allergy Immunol 2002;127:161-169.

57 Goldman DL, Huffnagle GB: Potential contribution of fungal infection and colonization to the development of allergy. Med Mycol 2009;47:445-456.

-58 Takenaka H, Ushio H, Niyonsaba F, Jayawardana ST, Hajime S, Ikeda S, Ogawa $\mathrm{H}$, Okumura K: Synergistic augmentation of inflammatory cytokine productions from murine mast cells by monomeric IgE and Tolllike receptor ligands. Biochem Biophys Res Commun 2010;391:471-476.

-59 Jayawardana ST, Ushio H, Niyonsaba F, Gondokaryono SP, Takenaka H, Ikeda S, Okumura K, Ogawa H: Monomeric IgE and lipopolysaccharide synergistically prevent mast-cell apoptosis. Biochem Biophys Res Commun 2008;365:137-142.

60 McCurdy JD, Olynych TJ, Maher LH, Marshall JS: Cutting edge: distinct Toll-like receptor 2 activators selectively induce different classes of mediator production from human mast cells. J Immunol 2003; 170: 1625-1629.

61 Kasakura K, Takahashi K, Aizawa T, Hosono A, Kaminogawa S: A TLR2 ligand suppresses allergic inflammatory reactions by acting directly on mast cells. Int Arch Allergy Immunol 2009;150:359-369.

62 Melendez AJ, Harnett MM, Pushparaj PN, Wong WS, Tay HK, McSharry CP, Harnett $\mathrm{W}$ : Inhibition of $\mathrm{Fc}$ epsilon RI-mediated mast cell responses by ES-62, a product of parasitic filarial nematodes. Nat Med 2007; 13:1375-1381.

63 Kimman TG, Terpstra GK, Daha MR, Westenbrink F: Pathogenesis of naturally acquired bovine respiratory syncytial virus infection in calves: evidence for the involvement of complement and mast cell mediators. Am J Vet Res 1989;50:694-700.

64 Marone G, Rossi FW, Detoraki A, Granata F, Marone G, Genovese A, Spadaro G: Role of superallergens in allergic disorders. Chem Immunol Allergy 2007;93:195-213. 